\title{
A HYBRID ALgORITHM BASED ON INVASIVE WEED OPTIMIZATION ALGORITHM AND GREY WOLF OPTIMIZATION ALGORITHM
}

\author{
Wisam Abdulelah Qasim ${ }^{1}$ and Ban Ahmed Mitras ${ }^{2}$ \\ ${ }^{1}$ M.sc. Student, Department of Mathematics, College of Computer Science \& \\ Mathematics, Mosul, Iraq. \\ ${ }^{2}$ Department of Mathematics, College of Computer Sciences \& Mathematics, \\ Mosul, Iraq.
}

\begin{abstract}
In this research, two algorithms first, considered to be one of hybrid algorithms. And it is algorithm represents invasive weed optimization. This algorithm is a random numerical algorithm and the second algorithm representing the grey wolves optimization. This algorithm is one of the algorithms of swarm intelligence in intelligent optimization. The algorithm of invasive weed optimization is inspired by nature as the weeds have colonial behavior and were introduced by Mehrabian and Lucas in 2006. Invasive weeds are a serious threat to cultivated plants because of their adaptability and are a threat to the overall planting process. The behavior of these weeds has been studied and applied in the invasive weed algorithm. The algorithm of grey wolves, which is considered as a swarm intelligence algorithm, has been used to reach the goal and reach the best solution. The algorithm was designed by SeyedaliMirijalili in 2014 and taking advantage of the intelligence of the squadrons is to avoid falling into local solutions so the new hybridization process between the previous algorithms GWO and IWO and we will symbolize the new algorithm IWOGWO.Comparing the suggested hybrid algorithm with the original algorithms it results were excellent. The optimum solution was found in most of test functions.
\end{abstract}

\section{KEYWORDS}

Invasive weeds optimization algorithm, grey wolves optimization algorithm, hybrid algorithms, optimization.

\section{INTRODUCTION}

Optimization is used in our daily lives (daily routine). The departments work to increase profits while reducing costs, engineering designer when designing the product works to maximize the performance of the product while working to reduce the cost at the same time. Recent years have seen the emergence of many complex optimization issues that are the reason for the development of highly efficient algorithms. Optimization suffers from several problems including:

1. There is chaos in evaluating the solution.

2. It is not easy to sort (distinguish) the optimal comprehensive solution from the local.

3. Problems related to matter constraints [1],[2].

The tendency used in the intuitive algorithms is one of the latest developments in the past two decades. Note that algorithms (intuitive or post-intuitive) make up the vast majority of modern techniques of optimization. 
We note that they have become very useful and powerful for solving complex optimization issues and have applied these algorithms in all important areas including agricultural services, engineering, science and in industrial applications as well [3].

The ways to solve optimization problems are two types:

1- Random algorithms.

2- Specific algorithms.

The specific algorithms represents most of the classical algorithms. The simplex method is a clear example of linear programming. It is a specific algorithm. We also observe the use of the derivative and are called derivative-based algorithms. One example is the Newton-Raphson algorithm, which relies on the derivative, which uses the values of the target function and its derivative, which we observe to behave well in smooth unimodal problems[2].

Methods of random algorithms:

1. Intuitive methods.

2. Post-Intuitive methods.

Glover was the first to introduce the term intuitive algorithm in 1986, which is the evolution of the intuitive algorithm[2].

The post-intuitive algorithm operates at a higher level than the intuitive algorithm.

We observe that all post-intuitive are used

1- Local Search.

2- Confirmed swap for random distribution[2].

It is noticeable that there is no agreed definition of methods (intuitive or post-intuitive) where the term (intuitive and post-intuitive) is used interchangeably and note that the recent trend refers to the designation of all random algorithms that use local research and random distribution intuitive algorithmic. Most intuitive algorithms are appropriate algorithms to solve global optimization matters[4].

The two important elements in the (post-intuitive) algorithm, which are considered important elements are: -

1- Intensification: It is intended to intensify research in the local area is done by using information that the current best solution can be available in this region and this corresponds to the principle of selecting the best ensures that the results will approach the optimization.

2- Diversification : is the generation of contradictory solutions for exploring the research space at the general level[2],[4].

The post intuitive can be classified in several methods. These methods can be classified depending on the path and society. Examples are the genetic algorithm, which is classified according to the community. A set of sections are used during the solution. The method of simulating steel is that it uses a single element that moves within the search space and the search method is a piece-piece that is the best solution or best move. It is usually acceptable and when the move is not good, it usually accepts a certain/ probability, since the movements affect the path in the search space and the probability of non-zero, the path up to a comprehensive optimization[4]. 
The problem of the research focused on obtaining the optimal comprehensive solution for the problems of unrestricted and high measurement and avoid falling into the problems of local solutions using the characteristics of the algorithm of swarms have been selected, one of the algorithms of the swarms algorithm grey wolves GWO. So as to avoid the emergence of local solutions[5].

The importance of the research is to develop a new way to solve the problems of optimization, which is a difficult issue, in order to benefit the designers of the proposed hybrid algorithm and any question falls within the difficult issues such as the issue of vehicle paths and many other issues.

The aim of the research is to suggestion a new algorithm called Hybrid Algorithm where the algorithm (invasive weeds optimization ) and the algorithm (grey wolves optimization) and the resulting algorithm IWOGWO is a hybrid algorithm to take advantage of the positive qualities and minimize the negatives of the previous two algorithms.

In recent years, hybrid algorithm has been introduced, knowing that designers have known it since the $1980 \mathrm{~s}$.

\section{INVASIVE WEED OPTIMIZATION ALgORITHM (IWO)}

It is an algorithm designed first by Mehrabian and Lucas in 2006 and the algorithm is inspired by biology (bio). It is a numerical optimization algorithm that simply simulates the natural behavior of weeds in colonization and finding a good place for growth and reproduction. Some of the characteristics of the IWO algorithm compared to other development algorithms. They are methods of reproduction, spatial dispersion and competitive exclusion[6].

The IWO algorithm includes a number of key steps that are applied to issues to find and improve the solution, and are usually interrelated as below:

\subsection{Steps of invasive weeds algorithm}

Setp1 Primary community:

A search area is taken and some number of weeds are randomly initialized in the full search space.

Step2 Reproduction:

Plants produce seeds based on the given fitness function. As well as the limits (upper-lower) of the function of decency in the colony. We observe that theNo. of seeds which the plant produce linearly increases from the min.probable seed producing to the max.[6].

$$
\operatorname{seed}_{i}=S_{\min }+\text { floor }\left(\frac{f_{i}-f_{\min }}{f_{\max }-f_{\min }}\left(S_{\max }-S_{\min }\right)\right)
$$

Equ.1 describe how to get seeds from the target function[7].

Step3 Spatial dispersion:

In this step randomly generated seeds are distributed over dimensions in the entire search space. By normal distribution at a rate of zero and a different variation (variable). 
International Journal of Artificial Intelligence and Applications (IJAIA), Vol.11, No.1, January 2020

This step means that the seeds are randomly distributed around the mother plant.

However, the standard deviation of the random function will decrease from a predetermined initial value $\left(\sigma_{\text {initial }}\right)$ to a final value $\left(\sigma_{\text {final }}\right)$ at each step as

$$
\sigma_{\text {iter }}=\frac{\left(\text { iter }_{\max }-\text { iter }\right)^{\mathrm{n}}}{\left(\text { iter }_{\max }\right)^{\mathrm{n}}}\left(\sigma_{\text {initial }}-\sigma_{\text {final }}\right)+\sigma_{\text {final }}
$$

To calculate the location of the new seeds, we use the following equation:

$$
\mathrm{X}_{\text {son }}=\mathrm{X}_{\text {parent }}+\mathrm{sd}=\mathrm{X}_{\text {parent }}+\operatorname{randn}(0,1) * \sigma_{\text {iter }}
$$

where

$\mathrm{X}_{\text {son }}$ represents the location of the sons.

Xparentrepresentsparents site.

Randn generate random numbers by normal distribution $(0,1)$ and record[8].

Step4 Competitive exclusion

Competition exists among plants for survival and if the plant is without offspring and its fate is extinct when $\mathrm{P}_{\max }$ reaches the maximum number of plants in the colony, the process of exclusion for a plant with poor fitness will begin.

\subsection{Exclusion mechanism:}

Upon reaching the maximum amount of herbs in the colony, each herb will be able to produce seeds and will then spread to the place of research. When all the seeds are concentrated in their position they are fitted with the mother plant (colony). Weeds with a low fitness function are excluded to reach the maximum acceptable to the community in the colony.

Note that plants and their offspring, as well as the element that has a high fitness function or (the best fitness function) will survive and will have a repetition in the algorithm [6].

\section{GREY WOLVESOPTIMIZATION ALGORITHM (GWO)}

This algorithm is based on the behavior of hunting and is considered this algorithm inspired by nature in the past years of great importance has helped accept this algorithm between designers and applied in different areas of life, because it is characterized by 1-Flexibility 2- Simplicity The GWO algorithm is one of the methods of optimization adopted swarm intelligence, which is within the social intelligence, which is based on supra-intuitive, which solves difficult problems.

It was designed by SeyedaliMirijalili in 2014 [9].

The levels of grey wolves are divided into four levels:

1) $\quad \alpha$ alpha 2) $\beta$ beta 3) $\delta$ Delta 4) $\omega$ omega The figure shows the hierarchy 


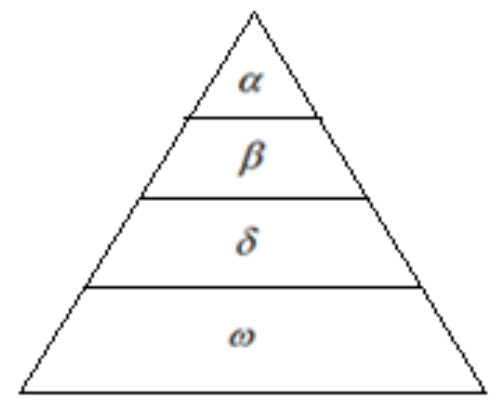

Figure 1. The hierarchy of the four levels [10].

The wolves $\alpha$ represent the leader of the group and are recognized by the elements of the group by fixing their tails. The alpha element is called (the mastermind). The only element $\alpha$ is allowed to mate in the group and the element $\alpha$ is responsible for decision-making and is also responsible for managing the group. The decisions he makes are 1-bedtime 2-fishing time 3-waking up time and many other decisions.

The strength of the grey wolves lies in the commands of the $\alpha$ commanding element by the group, which is evidence of the organization and discipline of the group[11],[12].

Followed by the element $\beta$ and come on in the second level after element $\alpha$. The element $\beta$ must respect the element $\alpha$. The element $\beta$ plays the role of the leader on lower-level elements of the group.The element $\beta$ is a consultant to the $\alpha$ and polite element of the group. At the third level come the wolves (element $\delta$ ), which work with $\beta$ to obey the group of element $\alpha$ and be superior to element $\omega$.The element $\omega$ offers obedience to all higher levels. The ram is considered a scapegoat for the group.

The element $\omega$ allows it to eat at the end after the elements of the higher-level group are finished. It may seem that its role is not important in the group, but when elements are lost, internal fighting and problems within the group are observed. This helps to satisfy the whole group and maintain the structure of hegemony [11],[12].

\subsection{Mathematical model}

The search is performed by the $\alpha$ element. Often unless research is assisted by elements $\beta$ and $\delta$, hunting may also be shared if required, but not always.

The first three solutions $\alpha, \beta$ and $\delta$ mimic the hunting behavior of the grey wolves, which are usually preserved. And got it yet.

Modeling in grey wolf optimization is the best solution. The element $\alpha$ is the best solution and then the element $\beta$ and then the element $\delta$ respectively. Finally the element omega. Grey wolves adopted the hunting technique based on $\alpha, \beta$, and $\delta$, and only the omega element followed[13].

The main hunting stages of grey wolves are:

1. Search for prey.

2. Encircle and bother the prey till it stops movement

3. Attack towards prey (hunting). 
First: Search for prey:

The prey hunt is first prepare the way for prey hunt through random configuration in the grey wolf search space [14].

Second: Encircling and harassing the prey

Equationsnumbered ( 4 and 5) represent the encircling behavior of grey wolves around prey through mathematical modeling. Using equations numbered (4 and 5), the elements update their position within the solution space around the prey.

$$
\begin{aligned}
& \overrightarrow{\mathrm{D}}=\left|\overrightarrow{\mathrm{C}} * \overrightarrow{\mathrm{X}}_{\mathrm{p}}-\overrightarrow{\mathrm{X}}(\mathrm{t})\right| \\
& \overrightarrow{\mathrm{X}}(\mathrm{t}+1)=\overrightarrow{\mathrm{X}}_{\mathrm{p}}-\overrightarrow{\mathrm{A}} * \overrightarrow{\mathrm{D}}
\end{aligned}
$$

$\vec{A}$ and $\vec{c}$ represent Vector coefficients are calculated as follows:

$$
\begin{aligned}
& \overrightarrow{\mathrm{A}}=2 * \overrightarrow{\mathrm{a}} * \mathrm{r}_{1}-\overrightarrow{\mathrm{a}} \\
& \overrightarrow{\mathrm{C}}=2 * \overrightarrow{\mathrm{r}}_{2}
\end{aligned}
$$

The values of $\vec{a}$ linearly decrease from 2 to 0 over the iterations

$r_{1}$ and $r_{2}$ are random vectorstracking standard uniform distribution $[0,1]$

Equation number (8) represents the updating of the parameter (a):

$$
\mathrm{a}=2-\mathrm{t}(2 / \mathrm{T})
$$

$\mathrm{t}$ : is the recent repetition.

$\mathrm{T}$ : is the greater number of repetition [10].

Using equations numbered (4 and 5), the grey wolf can update its position in the space around the prey and at any random location. Grey wolves will move around the best solution ever obtained [11].

Third: Attack towards prey (hunting)

Grey wolves have the potential to characterize prey and encircle them. After completing the encirclement, it focuses on hunting prey. The catch is usually by $\alpha$ and element $\beta$ and $\delta$ may be involved in the catch in the abstract search space. The best location of prey cannot be predicted. To simulate the hunting behavior we assume that $\alpha$ is the best candidate solution. Element $\beta$ and $\delta$ have a better knowledge of the potential location of prey. It can simulate the behavior of elements $\alpha, \beta$ and $\delta$ in the following formulas [15]:

$$
\overrightarrow{\mathrm{D}}_{\propto}=\left|\overrightarrow{\mathrm{C}}_{1} * \overrightarrow{\mathrm{X}}_{\propto}-\overrightarrow{\mathrm{X}}\right|
$$




$$
\begin{aligned}
& \overrightarrow{\mathrm{D}}_{\beta}=\left|\overrightarrow{\mathrm{C}}_{2} * \overrightarrow{\mathrm{X}}_{\beta}-\overrightarrow{\mathrm{X}}\right| \\
& \overrightarrow{\mathrm{D}}_{\delta}=\left|\overrightarrow{\mathrm{C}}_{3} * \overrightarrow{\mathrm{X}}_{\delta}-\overrightarrow{\mathrm{X}}\right|
\end{aligned}
$$

The following equations can be used to calculate the position vector of prey for wolves $\alpha, \beta$ and $\delta$.

$$
\begin{gathered}
\overrightarrow{\mathrm{X}}_{1}=\overrightarrow{\mathrm{X}}_{\alpha}-\overrightarrow{\mathrm{A}}_{1} * \overrightarrow{\mathrm{D}}_{\alpha} \\
\overrightarrow{\mathrm{X}}_{2}=\overrightarrow{\mathrm{X}}_{\beta}-\overrightarrow{\mathrm{A}}_{1} * \overrightarrow{\mathrm{D}}_{\beta} \\
\overrightarrow{\mathrm{X}}_{3}=\overrightarrow{\mathrm{X}}_{\delta}-\overrightarrow{\mathrm{A}}_{1} * \overrightarrow{\mathrm{D}}_{\delta}
\end{gathered}
$$

It is finding the rate for $\alpha, \beta$ and $\delta$.

The best position can be calculated as shown in formula (15).

$$
\left.\overrightarrow{\mathrm{X}}(\mathrm{t}+1)=\overrightarrow{\mathrm{X}}_{1}+\overrightarrow{\mathrm{X}}_{2}+\overrightarrow{\mathrm{X}}_{3}\right) / 3
$$

\subsection{The Grey Wolf Algorithm (Gwo)}

We can summarize the steps of the Grey Wolf Optimization Algorithm as shown below:

1- A random preparation for the population of the community of grey wolves.

2- Random configuration of parameters $\mathrm{A}, \mathrm{c}$ and $\mathrm{a}$.

3- find the fitness value.

4- For all iterations.

* We find the best search element is X $\alpha$.

* We find the second best search element $\mathrm{X} \beta$.

* We find the third search element $\mathrm{X} \delta$.

5- If the current iteration is less than the total iteration, then the research elements = Omega.

Equation 4 and 5 of the mathematical model are used

Otherwise, the search elements are $, \mathrm{x}_{\alpha}, \mathrm{x}_{\beta}, \mathrm{x}_{\delta}$

Equations 9 to 15 of the mathematical model are used

6- $\mathrm{i}=\mathrm{i}+1$

7- Return, $\mathrm{x}_{\alpha}$

8- the end

\subsection{Exploration And Exploitation}

The optimization algorithm may highlight a particular problem during the exploration process. We note that the algorithm tries to discover new areas of the search area for the problem by applying sudden changes in solutions and that the main goal is to discover the search space and not to fall into the best local position.

The main objective of exploitation is to approach the estimated optimal solutions achieved in the previous process (exploration). 
By discovering the neighborhood for each solution. Changes in solutions must be made and be gradual in order to conform to the optimal level.

The variable $\mathrm{C}$ is a parameter for the main control of the grey wolf optimization algorithm that enhances exploration. This parameter always returns the random value in the period [0,2] This contribution is usually strong at number [1]. Any greater than $\mathrm{C}$ note that the solution is attracted towards prey.

This parameter will provide random values and even that iteration number. Therefore, emphasis is placed on exploitation during improvement in the case of stagnation of the local situation.

The second parameter is A which is the control parameter that leads to exploration, where the value is defined based on a, decreasing linearly from 2 to 0 .

Because the random elements change their range within the period $[-2,2]$ in this parameter, this is for parameter A, where the exploration is strengthened when $\mathrm{A}$ is greater than one and $\mathrm{A}$ is smaller than one negative, i.e. at half of the iterations. While there is an emphasis on exploitation when A falls within the period $[-1,1]$ of the second half of the iterations, a good balance between exploitation and exploration may be required to find an accurate approximation of optimal global optimization.

So using random algorithms. This equilibrium occurs in the GWO algorithm, with the behavior of coefficient a decreasing in equation to obtain the vector A [13].

\section{HYBRID Algorithm}

Many researchers have developed algorithms through hybridization between common and difficult NP-Hard problems. Therefore, researchers have been working in the past years to hybridize algorithms with normal and difficult issues and have obtained the best results through the application of hybrid issues. The real purpose of the hybridization process is to obtain general and varied solutions that can deal with real world problems.

Hybrid algorithms have relied on swarm intelligence, a newly recognized branch of artificial intelligence that studies the collective behavior of complex self-regulating and decentralized systems. The term intelligence of the swarms emerged by the two researchers Jingwing and Geradobeni in 1989 as a set of algorithms and was used to control the swarms of robots[1].

In addition to the IWO optimization algorithm with GWO grey wolves algorithm to find a comprehensive solution to optimization issues.

\subsection{Hybrid Invasive Weed Algorithm Suggested}

The new hybrid algorithm has been named IWOGWO suggested.

The invasive weed optimization algorithm is distinct from other evolutionary algorithms including:

1 - reproduction. 2 - spatial dispersion. 3 - competitive exclusion.

The maximum benefit was achieved from the application of these properties in the process of hybridization. 
Steps of the IWOGWO hybrid algorithm

Step1 Preparingan initial population

The primary community is created by generating a primary community of solutions and calculating the value of the fitness function of that community.

Step2 use the gray wolves algorithm

Use the gray wolves algorithm to improve random initial population generated from step number (1) and we get improved population enters into step number (3).

\section{Step3 Re-producing}

New seeds can be obtained by equation (1), which allows obtaining new offspring.

\section{Step4 Spatial dispersion}

Seed propagation in the research space gives this algorithm the adaptability and randomness algorithm to use

1- Standard deviation. 2- Normal distribution.

And those calculated from equation numbered (2) and this allows the spread of seeds in the research space.

Step5 Locating the sons

It is determined by equation numbered (3) After parents and children are brought together, they will form a colony of weeds.

\section{Step6 Competitive exclusion}

After the order of the improved community comes the role on the property of competitive exclusion. Upon reaching the maximum allowable in the colony of (number of plants) Pmax then are excluded elements of low fitness and repeat the process until the optimal solution or until the condition is stop. 


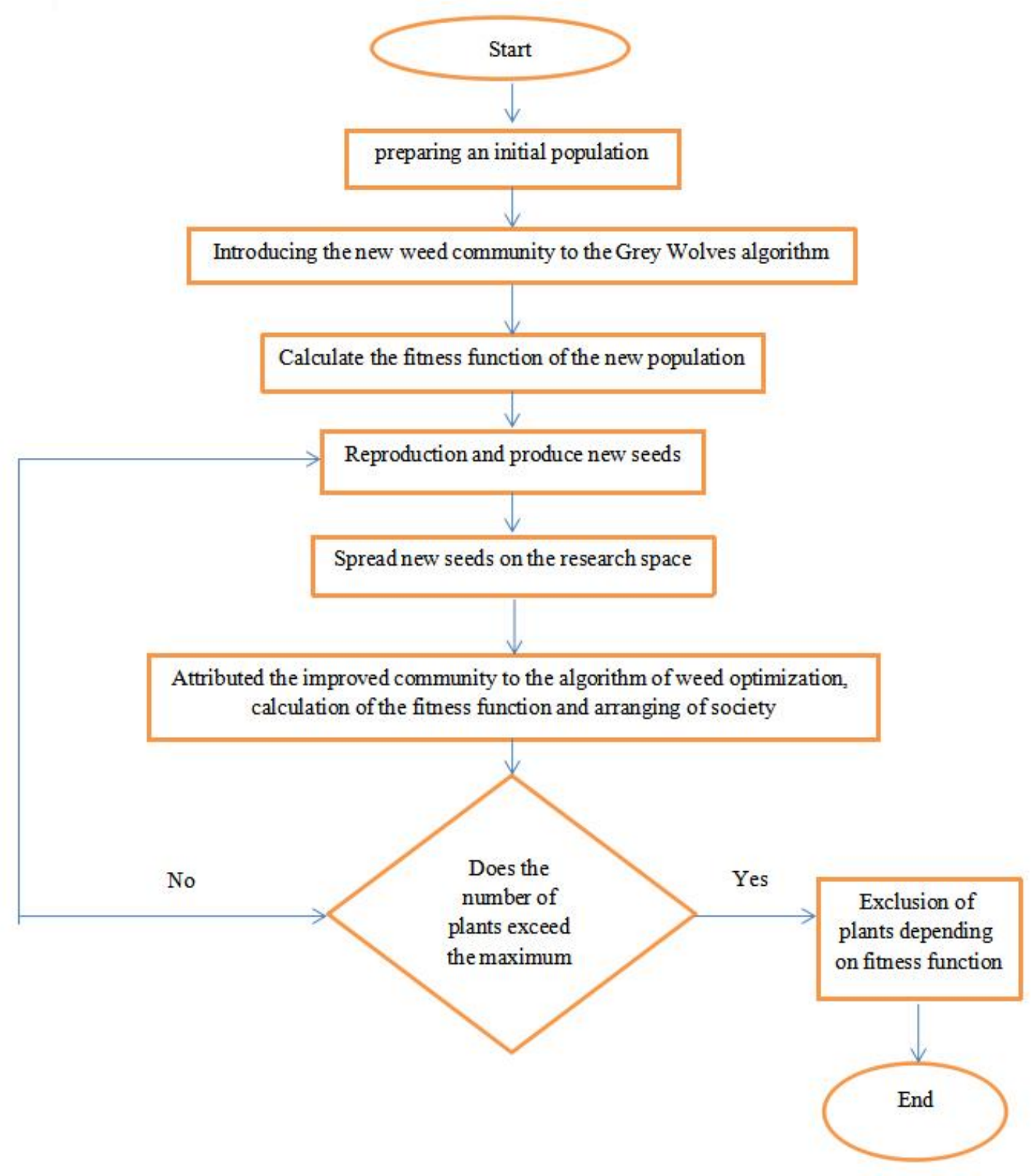

Figure 2. Flowchart of the Proposed IWOGWO Algorithm

\section{Practical Aspect}

The hybrid algorithm (IWOGWO) has been tested in terms of efficiency by using this algorithm to solve 10 a function of numerical optimization functions which is a highly efficient problems.

The IWIGWO hybrid algorithm is a combination of the algorithms of weed optimization and the algorithm of grey wolves.

The table below shows the initial parameters that must be specified before starting the program. 
International Journal of Artificial Intelligence and Applications (IJAIA), Vol.11, No.1, January 2020

Table1. Initial parameters

\begin{tabular}{|l|l|l|l|}
\hline Parameter & IWO & GWO & IWOGWO \\
\hline Npop0 & $5-10-15-20-25$ & $5-10-15-20-25$ & $5-10-15-20-25$ \\
\hline Npop & 25 & 25 & 25 \\
\hline N & 2 & --- & 2 \\
\hline Smin & 0 & --- & 0 \\
\hline Smax & 5 & --- & 5 \\
\hline MaxIt & 1000 & 1000 & 1000 \\
\hline Sigma initial & 0.5 & ---- & 0.5 \\
\hline Sigma final & 0.001 & --- & 0.001 \\
\hline
\end{tabular}

The laptop was used has the following specifications:

CPU speed is $2.4 \mathrm{GHz}$.

RAM size is $4 \mathrm{~GB}$

Matlab R2013a program works on windows7.

Table 2. Test functions

\begin{tabular}{|c|c|c|}
\hline Function & Range & Dim \\
\hline$F_{1}(x)=\sum_{i=1}^{n} x_{i}^{2}$ & {$[-100,100]$} & 30 \\
\hline$F_{2}(x)=\sum_{i=1}^{n}\left|x_{i}\right|+\prod_{i=1}^{n}\left|x_{i}\right|$ & {$[-10,10]$} & 30 \\
\hline $\mathrm{F}_{3}(\mathrm{x})=\sum_{\mathrm{i}=1}^{\mathrm{n}}\left(\sum_{\mathrm{j}-1}^{\mathrm{i}} \mathrm{x}_{\mathrm{i}}\right)^{2}$ & {$[-100,100]$} & 30 \\
\hline $\mathrm{F}_{4}(\mathrm{x})=\max _{\mathrm{i}}\left\{\left|\mathrm{x}_{\mathrm{i}}\right| .1 \leq \mathrm{i} \leq \mathrm{n}\right\}$ & {$[-100,100]$} & 30 \\
\hline$F_{5}(x)=\sum_{i=1}^{n}\left|x_{i}^{2}-10 \cos \left(2 \pi x_{i}\right)+10\right|$ & {$[-5,5]$} & 30 \\
\hline $\begin{aligned} & \mathrm{F}_{6}(\mathrm{x})=-20 \exp \left(-0.2 \sqrt{\frac{1}{\mathrm{n}} \sum_{\mathrm{i}=1}^{\mathrm{n}} \mathrm{x}_{\mathrm{i}}^{2}}\right) \\
&- \exp \left(\frac{1}{\mathrm{n}} \sum_{\mathrm{i}=1}^{\mathrm{n}} \cos \left(2 \pi \mathrm{x}_{\mathrm{i}}\right)\right)+20 \\
&+\mathrm{e}\end{aligned}$ & {$[-500,500]$} & 30 \\
\hline$F_{7}(x)=\frac{1}{4000} \sum_{i=1}^{n} x_{i}^{2}-\prod_{i=1}^{n} \cos \left(\frac{x_{i}}{\sqrt{i}}\right)-1$ & {$[-0.5,0.5]$} & 30 \\
\hline
\end{tabular}


International Journal of Artificial Intelligence and Applications (IJAIA), Vol.11, No.1, January 2020

\begin{tabular}{|c|c|c|}
\hline $\begin{aligned} & \mathrm{F}_{8}(\mathrm{x})=\frac{\pi}{\mathrm{n}}(10 \sin \left(\pi \mathrm{y}_{\mathrm{i}}-1\right)^{2}[1 \\
&+\left.10 \sin ^{2}\left(\pi \mathrm{y}_{\mathrm{i}+1}\right)\right]+\left(\mathrm{y}_{\mathrm{n}-1}\right)^{2} \\
&+\sum_{\mathrm{i}=1}^{\mathrm{n}} \mathrm{U}\left(\mathrm{x}_{\mathrm{i}}, 10,100,4\right) \\
& \mathrm{y}_{\mathrm{i}}=1+\frac{\mathrm{X}_{\mathrm{i}+1}}{4} \mathrm{u}\left(\mathrm{x}_{\mathrm{i}}, \mathrm{a}, \mathrm{k}, \mathrm{m}\right) \\
&=\left\{\begin{array}{c}\mathrm{k}\left(\mathrm{x}_{\mathrm{i}}-\mathrm{a}\right)^{\mathrm{m}} \mathrm{x}_{\mathrm{i}}>0 \\
0-\mathrm{a}<\mathrm{x}_{\mathrm{i}}<a \\
\mathrm{k}\left(-\mathrm{x}_{\mathrm{i}}-\mathrm{a}\right)^{\mathrm{m}} \mathrm{x}_{\mathrm{i}}<-a\end{array}\right.\end{aligned}$ & [-pi, pi] & 30 \\
\hline $\mathrm{F}_{9}(\mathrm{x})=\sum_{\mathrm{i}=1}^{11}\left|\mathrm{a}_{\mathrm{i}}-\frac{\mathrm{x}_{\mathrm{i}}\left(\mathrm{b}_{\mathrm{i}}^{2}+\mathrm{b}_{\mathrm{i}} \mathrm{x}_{2}\right)}{\mathrm{b}_{\mathrm{i}}^{2}+\mathrm{b}_{\mathrm{i}} \mathrm{x}_{2}+\mathrm{x}_{4}}\right|^{2}$ & {$[-15,15]$} & 4 \\
\hline $\begin{array}{c}\mathrm{F}_{10}(\mathrm{x})=4 \mathrm{x}_{\mathrm{i}}^{2}-2.1 \mathrm{x}_{1}^{4}+\frac{1}{3} \mathrm{x}_{1}^{6}+\mathrm{x}_{1} \mathrm{x}_{2}-4 \mathrm{x}_{2}^{2} \\
+4 \mathrm{x}_{2}^{4}\end{array}$ & {$[-5,5]$} & 2 \\
\hline
\end{tabular}

Table 3. results of the algorithm IWO and GWO and compared with the results of hybrid algorithm IWOGWO by using 5 elements and 1000 iterations.

\begin{tabular}{|l|l|l|l|}
\hline Function & IWO & GWO & IWOGWO \\
\hline F1 & $3.48348 * 10-5$ & $1.727891 * 10-22$ & 0 \\
\hline F2 & 0.1421731 & $12.8631 * 10-14$ & 0 \\
\hline F3 & $3.847 * 10-4$ & $3.36267 * 10-2$ & 0 \\
\hline F4 & 31.06199 & $21.81783 * 10-6$ & 0 \\
\hline F5 & 121.29128 & $9.8558 * 10-1$ & 0 \\
\hline F6 & 19.10598 & $3.83533 * 10-12$ & $4.4409 * 10-15$ \\
\hline F7 & $9.8396308 * 10-3$ & $1.09755 * 10-2$ & 0 \\
\hline F8 & 25.26338 & 0.236355 & 0.63752 \\
\hline F9 & $3.9978 * 10-4$ & $2.5659 * 10-3$ & 0.10027 \\
\hline F10 & -1.0316 & -1.0316 & -0.99311 \\
\hline
\end{tabular}

Table4. results of the algorithm IWO and GWO and compared with the results of hybrid algorithm IWOGWO by using 10 elements and 1000 iterations.

\begin{tabular}{|l|l|l|l|}
\hline Function & IWO & GWO & IWOGWO \\
\hline F1 & $3.38112 * 10-5$ & $4.489823 * 10-35$ & 0 \\
\hline F2 & 0.0247183 & $2.176478 * 10-21$ & 0 \\
\hline F3 & $1.91002 * 10-3$ & $6.74407 * 10-7$ & 0 \\
\hline F4 & 32.73155 & $70.59953 * 10-8$ & 0 \\
\hline F5 & 85.77135 & 1.93722000 & 0 \\
\hline F6 & 19.1387 & $3.81918 * 10-14$ & $7.9936 * 10-15$ \\
\hline F7 & $8.865911^{*} 10-2$ & $4.0325 * 10-3$ & 0 \\
\hline F8 & 19.86204 & $8.82853 * 10-2$ & 0.23232 \\
\hline F9 & $2.45339 * 10-3$ & $4.406469 * 10-3$ & 0.028729 \\
\hline F10 & -1.0316 & -1.0316 & -0.13653 \\
\hline
\end{tabular}


International Journal of Artificial Intelligence and Applications (IJAIA), Vol.11, No.1, January 2020

Table5. results of the algorithm IWO and GWO and compared with the results of hybrid algorithm IWOGWO by using 15 elements and 1000 iterations.

\begin{tabular}{|l|l|l|l|}
\hline Function & IWO & GWO & IWOGWO \\
\hline F1 & $3.31392 * 10-5$ & $32.76772 * 10-44$ & 0 \\
\hline F2 & 0.0252103 & $6.00473 * 10-26$ & 0 \\
\hline F3 & $1.786933 * 10-4$ & $2.4346657 * 10-9$ & 0 \\
\hline F4 & $2.62742 * 10-3$ & $7.18576 * 10-11$ & 0 \\
\hline F5 & 76.22018 & 0.21267000 & 0 \\
\hline F6 & 19.07697 & $2.71782 * 10-14$ & $7.9936 * 10-15$ \\
\hline F7 & $1.377530 * 10-2$ & $3.9167 * 10-3$ & 0 \\
\hline F8 & 18.07077 & $7.62111 * 10-2$ & 0.77268 \\
\hline F9 & $7.67408 * 10-4$ & $4.57768 * 10-4$ & 0.030296 \\
\hline F10 & -1.0316 & -1.0316 & -1.0053 \\
\hline
\end{tabular}

Table6. results of the algorithm IWO and GWO and compared with the results of hybrid algorithm IWOGWO by using 20 elements and 1000 iterations.

\begin{tabular}{|l|l|l|l|}
\hline Function & IWO & GWO & IWOGWO \\
\hline F1 & $3.31477 * 10-5$ & $8.20779 * 10-50$ & 0 \\
\hline F2 & 0.026135 & $1.531945 * 10-29$ & 0 \\
\hline F3 & $1.367628 * 10-3$ & $5.4470181 * 10-13$ & 0 \\
\hline F4 & $2.48269 * 10-3$ & $96.194778 * 10-12$ & 0 \\
\hline F5 & 89.4533 & 0.3569500 & 0 \\
\hline F6 & 18.8478 & $2.29148 * 10-14$ & $7.9936 * 10-15$ \\
\hline F7 & $1.74733 * 10-2$ & $3.3852 * 10-3$ & 0 \\
\hline F8 & 18.44998 & $5.25017 * 10-2$ & 0.47124 \\
\hline F9 & $4.7212 * 10-4$ & $8.33160 * 10-3$ & 0.20087 \\
\hline F10 & -1.0316 & -1.0316 & -0.99192 \\
\hline
\end{tabular}

Table7. results of the algorithm IWO and GWO and compared with the results of hybrid algorithm IWOGWO by using 25 elements and 1000 iterations.

\begin{tabular}{|l|l|l|l|}
\hline Function & IWO & GWO & IWOGWO \\
\hline F1 & 298.640611 & $84.03248^{*} 10-56$ & 0 \\
\hline F2 & 0.0353479 & $1.490545^{*} 10-32$ & 0 \\
\hline F3 & 5924.11338 & $\begin{array}{l}752.383373 * 10- \\
15\end{array}$ & 0 \\
\hline F4 & 38.67039 & $96.73922 * 10-13$ & 0 \\
\hline F5 & 96.32194 & $1.13686 * 10-14$ & 0 \\
\hline F6 & 18.93683 & $1.68753 * 10-14$ & $1.1546 * 10-14$ \\
\hline F7 & 423.89807 & 0 & 0 \\
\hline F8 & 19.1291 & $6.605576 * 10-2$ & 0.39953 \\
\hline F9 & $6.97397 * 10-4$ & $6.335574 * 10-3$ & 0.050696 \\
\hline F10 & -1.0316 & -1.0316 & -0.88631 \\
\hline
\end{tabular}




\section{Conclusions}

In this research we have studied the algorithm of weed optimization and we have seen poor performance. This algorithm in terms of reaching the local micro point and this indicates a divergence from the optimal solution and to improve the performance. The original wolves algorithm is accessible to the local micro point and moves away from the optimal solution. As for the hybrid algorithm that avoids falling into the local IGA $\backslash \mathrm{d}$. The swarm algorithm had a great impact on improving the IWO algorithm and for this the new hybrid algorithm was proposed IWOGWO.We have obtained very good results. The overall optimal solution has been reached for most (10) test functions. This is what our results indicate. In the future, the authors plan to integrate the proposed algorithm IWO with another algorithm that depended on the intelligent swarm. Such integration will improve the performance to get more accurate results.

\section{REFERENCE}

[1] K. E. Parsopoulos and M. N. Vrahatis, "Particle swarm optimization and intelligence: advances and applications," 2010.

[2] H. T. Yaseen, B. A. Mitras, and A. S. M. Khidhir, "Hybrid Invasive Weed Optimization Algorithm with Chicken Swarm Optimization Algorithm to solve Global Optimization Problems," Int. J. Comput. Networks Commun. Secur., vol. 6, no. 8, pp. 173-181, 2018.

[3] X.-S. Yang, Engineering optimization: an introduction with metaheuristic applications. John Wiley \& Sons, 2010.

[4] X.-S. Yang, Nature-inspired metaheuristic algorithms. Luniver press, 2010.

[5] C. Blum, A. Roli, and M. Sampels, Hybrid metaheuristics: an emerging approach to optimization, vol. 114. Springer, 2008.

[6] A. R. Mehrabian and C. Lucas, "A novel numerical optimization algorithm inspired from weed colonization,” Ecol. Inform., vol. 1, no. 4, pp. 355-366, 2006.

[7] Y. Zhao, L. Leng, Z. Qian, and W. Wang, "A discrete hybrid invasive weed optimization algorithm for the capacitated vehicle routing problem," Procedia Comput. Sci., vol. 91, pp. 978-987, 2016.

[8] C. Liu and $\mathrm{H}$. Wu, "Synthesis of thinned array with side lobe levels reduction using improved binary invasive weed optimization,” Prog. Electromagn. Res., vol. 37, pp. 21-30, 2014.

[9] L. Korayem, M. Khorsid, and S. S. Kassem, "Using grey wolf algorithm to solve the capacitated vehicle routing problem," in IOP conference series: materials science and engineering, 2015, vol. 83, no. 1, p. 12014.

[10] Y. Ren, T. Ye, M. Huang, and S. Feng, "Gray Wolf Optimization Algorithm for Multi-Constraints Second-Order Stochastic Dominance Portfolio Optimization,” Algorithms, vol. 11, no. 5, p. 72, 2018.

[11] M. Karimi and S. M. Babamir, "QoS-aware web service composition using Gray Wolf Optimizer," Int. J. Inf. Commun. Technol. Res., vol. 9, no. 1, pp. 9-16, 2017.

[12] N. Singh and S. B. Singh, "Hybrid algorithm of particle swarm optimization and grey wolf optimizer for improving convergence performance,” J. Appl. Math., vol. 2017, 2017.

[13] H. Faris, I. Aljarah, M. A. Al-Betar, and S. Mirjalili, "Grey wolf optimizer: a review of recent variants and applications," Neural Comput. Appl., vol. 30, no. 2, pp. 413-435, 2018.

[14] H. Turabieh, "A Hybrid ANN-GWO Algorithm for Prediction of Heart Disease," Am. J. Oper. Res., vol. 6, no. 02, p. 136, 2016.

[15] S. Mirjalili, S. M. Mirjalili, and A. Lewis, “Grey Wolf Optimizer," Adv. Eng. Softw., vol. 69, pp. 4661,2014 